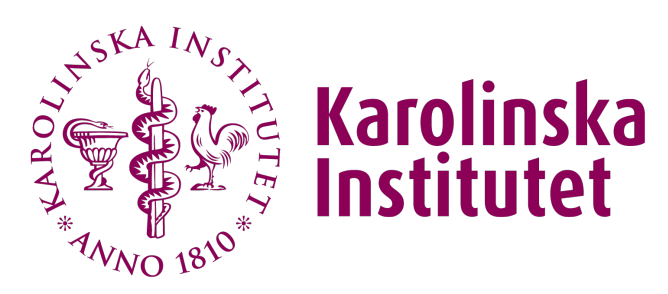

Karolinska Institutet

http://openarchive.ki.se

This is a Peer Reviewed Accepted version of the following article, accepted for publication in Tumor Biology.

2015-02-09

\title{
Novel and emerging targeted-based cancer therapy agents and methods
}

Hojjat-Farsangi, Mohammad

Tumour Biol. 2015 Feb;36(2):543-56.

http://doi.org/10.1007/s13277-015-3184-x

http://hdl.handle.net/10616/44518

If not otherwise stated by the Publisher's Terms and conditions, the manuscript is deposited under the terms of the Creative Commons Attribution-NonCommercial-NoDerivatives License (http://creativecommons.org/licenses/by-nc-nd/4.0/), which permits non-commercial re-use, distribution, and reproduction in any medium, provided the original work is properly cited, and is not altered, transformed, or built upon in any way. 


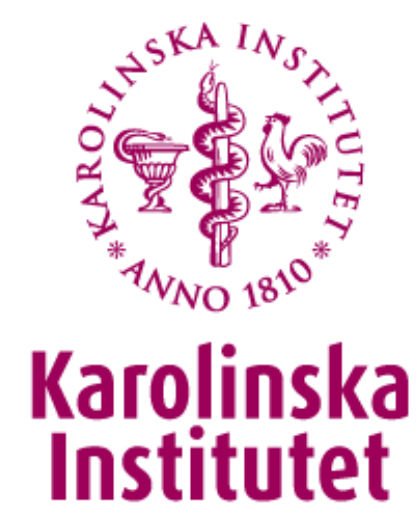

This is an author produced version of a paper accepted by Tumor Biology. This paper has been peer-reviewed but does not include the final publisher proof-corrections or journal pagination.

\section{Novel and emerging targeted-based cancer therapy agents and methods}

\section{Mohammad Hojjat-Farsangi}

The final publication is available at Springer via http://dx.doi.org/10.1007/s13277-015-3184-x 


\section{Novel and emerging targeted-based cancer therapy agents and methods}

\section{Mohammad Hojjat-Farsangi}

Department of Oncology-Pathology, Immune and Gene therapy Lab, Cancer Center Karolinska (CCK), Karolinska University Hospital Solna and Karolinska Institute, Stockholm, Sweden,

Department of Immunology, School of Medicine, Bushehr University of Medical Sciences, Bushehr, Iran

\section{Corresponding author:}

Mohammad Hojjat-Farsangi, Ph.D

Assistant Professor

Department of Oncology-Pathology

Cancer Center Karolinska (CCK)

Karolinska University Hospital, Solna

SE-171 76 Stockholm, Sweden

Tel: +46851774308

Fax: +468318327

Email: mohammad.hojat-farsangi@Ki.se

Key Words: targeted therapy, monoclonal antibody, small-molecule Inhibitor, tyrosine kinase, vaccine

Running title: Targeted-based cancer therapy agents and methods 


\begin{abstract}
After several decades of uncovering the cancer features and following the improvement of therapeutic agents; however cancer remains as one of the major reasons of mortality. Chemotherapy is one of the main treatment options and has significantly improved the overall survival of cancer patients, but these agents are highly toxic for normal cells. Therefore, there is a great unmet medical need to develop new therapeutic principles and agents. Targetedbased cancer therapy (TBCT) agents and methods have revolutionized the cancer treatment efficacy. Monoclonal antibodies (mAbs) and small molecule inhibitors (SMIs) are among the most effective agents of TBCT. These drugs have improved the prognosis and survival of cancer patients; however, the therapeutic resistance has subdued the effects. Several mechanisms lead to drug resistance such as mutations in the drug targets, activation of compensatory pathways and intrinsic or acquired resistance of cancer stem cells. Therefore, new modalities, improving current generation of inhibitors and mAbs as well as optimizing the combinational therapy regimens are necessary to decrease the current obstacles in front of TBCT. Moreover, the success of new TBCT agents such as mAbs, SMIs and immunomodulatory agents has sparked further therapeutic modalities with novel targets to inhibit. Due to the lack of cumulative information describing different agents and methods of TBCT, this review focuses on the most important agents and methods of TBCT that are currently under investigation.
\end{abstract}




\section{Introduction}

Cancer is a complex invasive disorder and is one of the major reasons of a significant mortality rate worldwide. Cancer incidence is correlated with a combination of the interaction of oncogenes, tumor suppressor gene mutations and environmental forces [1].

For several years, traditional chemotherapy has been the main treatment modality in cancer patients in addition to radiation therapy and surgery [2]. These agents and methods may lead to complete remission and be effective in reducing tumor size and metastasis. However, most chemotherapy agents kill dividing cancer and normal cells and have high incidence of lifethreatening complications [2]. On the other hand, resistance to chemotherapy presented a major obstacle to attempt to increase the prognosis of patients. Tumor cell resistance (intrinsic and acquired) results from the genetic and epigenetic modifications occurring in cancer cells before or after chemotherapy.

Therefore, developing new therapeutic agents and methods that specifically kill tumor cells, spare normal cells and overcome drug resistance is imminent.

Targeted-based cancer therapies (TBCT) have significantly improved and several specific agents and interesting approaches have been developed (Table 1) [3-8]. Moreover, the application of immunomodulatory (IMiDs) agents has tremendously improved the survival of cancer patients.

Among several TBCT drugs, different types of inhibitors such as small molecule inhibitors (SMIs), monoclonal antibodies (mAbs) and antagonists have been described to control the progression of various cancers $[5,9,10]$.

Targeting tumor cells using mAbs and SMIs against receptor tyrosine kinases (RTKs) or intracellular kinases have been described in several review articles [5, 9, 11]. This review describes the most important agents and methods of TBCT and the recent advances in the field of targeted cancer therapy.

\section{Small molecule inhibitors (SMIs)}

SMIs are chemical substances that interrupt with molecules required for cells growth and function. These agents specifically target molecules with a unique construction that differs from traditional chemotherapy drugs. SMIs are used for the treatment of various diseases such as autoimmune and malignant disorders $[5,12]$. 
Currently, several inhibitors are in clinical use or are under investigation in pre-clinical and clinical stages. SMIs of tyrosine kinases (tyrosine kinase inhibitors; TKIs) are one of the major groups.

Afatinib, erlotinib, lapatinib, ibrutinib, and sunitinib are examples among the current approved TKIs for cancer treatment. Moreover, new SMIs targeting RTKs such as AXL and ROR1 are promising drugs that are in pre-clinical settings [5, 9, 13].

Recently, several new and interesting inhibitors have emerged and will be discussed in following sections.

\section{Inhibitors of pro-survival signaling pathways}

Several inhibitors have been developed to target the intracellular key proteins, in which most of them are dysregulated pro-survival or signaling molecules. Upregulation of pro-survival modulators as well as suppression of anti-apoptotic proteins are important for tumor cells survival. Targeting these molecules such as Bcl family members involved in cell survival signaling pathways are of great importance.

\section{Pro-survival inhibitors}

Navitoclax (ABT-263) is a Bcl-2/Bcl-XL/Bcl-w inhibitor that binds to Bcl-2 family proteins with higher affinity than other Bcl-2 inhibitors (100-1000 fold greater). Bcl-XL is highly expressed on platelets and navitoclax induced thrombocytopenia in treated patients [14]. Significant clinical benefit has been demonstrated in chronic lymphocytic leukemia (CLL) patients [15]. Navitoclax induced partial remission in one-third of relapsed CLL patients. Preclinical and clinical studies have shown that navitoclax may enhance sensitivity of small cell lung cancer cells to standard cytotoxic agents $[15,16]$. Moreover, combination of TKIs with pro-survival inhibitors, such as navitoclax might also sensitize tumor cells to treatment [17]. Navitoclax is under investigation in combination with mAbs (e.g. rituximab), TKIs (e.g. erlotinib) and other drugs in clinical trials. Leukemic cells in the bone marrow (BM) are less responsive to navitoclax due to the contact with stromal cells and upregulation of antiapoptotic proteins [18]. Therefore, combination of other agents that release leukemic cells from BM or lymph nodes might increase the efficiency of navitoclax. Combination of navitoclax and ibrutinib may be an appropriate strategy to target resident tumor cells in tissues. Treatment of CLL patients with ibrutinib increased the number of blood lymphocyte and resulted in lymphocytosis. A majority of these CLL cells released from lymph nodes 
followed by rapid resolution of enlarged lymph nodes [19]. Released leukemic cells loss their contact with supporting stromal cells and become deprived of survival contacts [19].

\section{PARP inhibitors as part of DNA-repair machinery}

Poly ADP ribose polymerases (PARP) have been known as an important DNA repair enzyme group. These enzymes are present in the nucleus and are activated by DNA damage. Due to the crucial role of PARP enzymes, PARP inhibitors are potential and novel therapeutic drugs for cancer treatment.

Several PARP inhibitors are under investigation as single agents or in combination with other DNA damaging drugs such as ionizing radiation. Currently, more than nine PARP inhibitors are in different stages of clinical settings for cancer treatment (Table 2).

PARP inhibitors are more proper for the treatment of patients with mutated $B R C A 1 / 2$ (breast cancer, early onset) genes associated cancer than others. These mutations cause mistakes in DNA repair machinery and are lethal for cells when the DNA repair protein, PARP1 is inhibited [20].

Rucaparib (PF-01367338, AG-014699) is a PARP inhibitor, and pre-clinical studies have shown a better effect in combination with temozolomide [21]. In the first phase I trial, rucaparib combination with temozolomide was evaluated in 32 patients with different solid tumors [22]. Rucaparib combination with temozolomide showed PARP inhibition at all doses and in a dose escalation evaluation, PARP inhibitory dose was determined to be $12 \mathrm{mg} / \mathrm{m}^{2}$ with a constant dose of temozolomide at $100 \mathrm{mg} / \mathrm{m}^{2} /$ day. The maximal tolerated dose for the combination was $12 \mathrm{mg} / \mathrm{m}^{2}$ for rucaparib and $200 \mathrm{mg} / \mathrm{m}^{2} /$ day for temozolomide. Mean of PARP inhibition at 5 hours was determined to be $92 \%$, ranged from $46 \%$ to $97 \%$ and DNA single-strand breaks was noted for all treated patients. No major side effect was observed for rucaparib alone and no interaction with temozolomide was noted [22].

In a phase II study of the rucaparib, the combination with temozolomide in patients with metastatic melanoma was studied [23]. In this study, patients with no prior chemotherapy were evaluated. Treatment was given until disease progression. The response rate, median time to progression and median overall survival were $17.4 \%, 3.5$ and 9.9 months, respectively. Myelosuppression was described in 54\% of patients [23]. 
Olaparib or AZD-2281 is an inhibitor of PARP1/2 with peak plasma concentration between 13 hours and half-life of 5-7 hours. In the first-in-human phase I trial the maximal tolerated dose was established as $400 \mathrm{mg} / 2$ days [24]. Overall response rate and disease control rate were shown to be $47 \%$ and $63 \%$, respectively in 19 ovarian breast, or prostate patients with $B R C A$ gene mutations [24]. In several clinical trials olaparib has shown clinical benefits with anti-tumor activity in BRCA1 and 2- deficient breast and ovarian cancer patients [24].

Veliparib (ABT-888), iniparib (BSI-201), CEP-9722, E7016 (GPI-21016), INO-1001, and LT-673 (BMN-673) are other potent PARP inhibitors that are under investigation in clinical trials as single agent or in combination therapy.

\section{HDAC inhibitors}

Normal cellular functions such as cell cycle arrest at different stages and apoptosis are mostly regulated by histone proteins that are modulated by protein acetylation [25]. Deregulation of histones acetylation have been shown to be related with aggressive disease and poorer response to the current treatments [26]. The acetylation states of proteins are modified by the opposing effects of histone acetyl-transferases (HATs) and histone deacetylases (HDACs) [27].

HDACs are categorized into several classes based on homology to yeast HDACs and their dependence to Zinc. These groups are class I (HDACs 1-3 and 8) (also named true HDACs), class II a/b (HDACs 4-7,9 and 10) and class IV (HDAC 11) [25]. In contrast to HDAC class I members that are located in nucleus, class II HDACs are located in cytoplasm, but can translocate into the nucleus. Class III HDACs [sirtuin enzymes (SIRT 1-7)] are independent of Zinc for function. Moreover, HDAC classes have different histone substrates. Histone is the main substrate of class I while both histone and non-histone proteins are class II HDACs substrates, and conversely, non-histone proteins act as class III HDACs substrates [25].

Moreover, based on the chemical structure, HDACs inhibitors are classified into several groups. These groups are hydroxamic acids (trichostatin A), carboxylic acids (valproate), aminobenzamides (entinostat), cyclic peptides (apicidin), epoxyketones (trapoxins), and hybrid molecules [28].

Protein acetylation and deacetylation are dysregulated in several tumors, including breast, ovarian, pancreatic cancers, multiple myeloma, T-cell lymphoma (TCL), cutaneous T-cell 
lymphoma (CTCL), melanoma, neuroendocrine tumors, leukemias, and Hodgkin lymphoma [26]. HDACs inhibitors induce apoptosis, senescence, differentiation, and inhibit tumor cells angiogenesis and growth; however they have no major effects on normal cells.

Clinical evidences demonstrated that HDACs inhibitors have promising anti-tumor effects. Vorinostat (Zolinza), panobinostat (LBH-589), belinostat (PXD-101), entinostat (MS-275 or SNDX-275), mocetinostat (MGCD0103), and romidepsin (Istodax) are promising HDACs inhibitors and target different members of HDACs [25].

Vorinostat and romidepsin have been approved by the FDA for the treatment of patients with refractory CTCL [28]. Vorinostat was the first HDACs inhibitor approved by the FDA for the treatment of progressive CTCL on October 6, 2006 (Table 2) [29]. Phase II clinical trials for evaluation of romidepsin were started in 1997 on various malignancies and promising results were found in the treatment of CTCL and other peripheral T-cell lymphomas. On November 5, 2009, the FDA approved romidepsin for the treatment of CTCL [30].

Currently, new generation of HDACs inhibitors have been developed and some of them have entered the clinical trials, including CHR-3966, chidamide [31], AR-42, hydroxamides quisinostat, and abexinostat [28, 32]. Pre-clinical studies indicated these inhibitors are more potent than the parental agents, with proper pharmacodynamic, pharmacokinetic and lower side effects.

\section{MTOR inhibitors}

MTOR (mammalian target of rapamycin), also recognized as FK506 binding protein 12rapamycin associated protein 1 (FRAP1) belongs to the phosphatidylinositide 3-kinases (PI3K) protein family. MTOR is an intracellular serine-threonine kinase that collects the growth and survival signals received by tumor cells as a central kinase. It is activated in tumor cells by different mechanisms such as RTKs stimulation, oncogenes and loss of tumor suppressor genes [33].

Different mTOR inhibitors such as deforolimus, everolimus and temsirolimus have been approved for cancer treatment and several other inhibitors are in pre-clinical and clinical stages (Table 2). 
Deforolimus (ridaforolimus, AP23573 or MK-8669) is an analog of rapamycin. MTOR blocking by deforolimus induced a starvation effect in tumor cells by interfering with cell growth, cell division, metabolism, and angiogenesis [34]. Everolimus in combination with tamoxifen, letrozole, or exemestane has shown high clinical efficacy for the treatment of $\mathrm{ER}^{+}$ metastatic breast cancer patients [35]. This inhibitor was approved by the FDA for the treatment of advanced recurrent colorectal carcinoma after failure of the treatment with sunitinib or sorafenib [36]. On August 29, 2012, the FDA granted accelerated approval for everolimus for the treatment of patients with tuberous sclerosis complex who have subependymal giant cell astrocytoma (SEGA). Everolimus is the first pediatric inhibitor drug to be approved by the FDA for the treatment of tumors that occur primarily during childhood [37].

Temsirolimus (Torisel) is a derivative of sirolimus and was approved by the FDA and the European Medicines Agency (EMA) in May and November 2007, respectively for the treatment of patients with recurrent colorectal carcinoma [38]. It interferes with protein synthesis and controls tumor cells proliferation, growth and survival. Temsirolimus has been shown to induce cell cycle arrest in the G1 phase and prevented tumor angiogenesis by inhibiting VEGF synthesis [39].

It has been shown that the PI3K/Akt/mTOR pathway is used by $\mathrm{ER}^{+} / \mathrm{HER} 2^{+}$tumors to escape control of anti-ER and HER2 therapies, including, specific mAbs and SMIs. The combination of mTOR inhibitors with current ER/HER2-targeted therapies may be a promising approach for overcoming and preventing the development of drug resistance [40].

\section{Targeting RNA translation in tumor cells}

Several molecules involved in the process of RNA translation and protein synthesis are proper targets for special type of inhibitors that react with nucleic acids. RNA targeting is a developing approach to anti-tumor therapeutics that requires identification of specific inhibitors to target different RNA structures. Specific structures in RNA form several types of secondary structures like hairpin loops, internal loops, and bulged regions that are proper for the binding of inhibitors $[6,41]$.

Pre-mRNA splicing is an essential step in gene expression and the maintenance of highfidelity of this process is vital to allow correct protein expression [42]. MRNA splicing is 
usually disrupted in cancer that might be due to altered expression of RNA-binding proteins, involved in mRNA splicing and results in changes in normal process of alternatively spliced mRNAs [43]. Inhibitors or regulators that block or modify the splicing process of pre-mRNA might be proper for therapeutic applications. Currently, a few inhibitors are available with which to dissect the splicing process. Therefore, the identification of selective inhibitors that either prevent or change, pre-mRNA splicing would be valuable for therapeutic applications [43].

Polyamines are poly-cationic amines that play important roles in sustaining cellular growth and activities. In cancer cells, their concentration is high and decrease in concentration inhibits cellular growth and induces apoptosis [41]. Polyamines and analogues (e.g. 1naphthylacetyl spermine, NASPM) have been shown to interact and stabilize DNA and RNA. Some analogues have demonstrated strong activity against tumor growth in different types of cell lines [44]. Polyamine analogues do not substitute for the natural polyamines involved in normal cell function, therefore, they show selective anti-tumor activity [45]. Hence, polyamines are essential for cancer cells proliferation and targeting these agents is a proper strategy.

Moreover, several natural compounds, and their synthetic derivatives were described to prevent splicing. GEX1A, FR901464, E7107, pladienolide B, pladienolide D, sudemycin, and spliceostatin A (SSA) are examples of these compounds that target the SF3b subunit of the U2snRNP [43].

Madrasin is one of the mRNA splicing modulators that was reported by Pawellek et al. [43]. This inhibitor interfered with the early stages of spliceosome assembly and interrupts its assembly at the complex A. Madrasin is cytotoxic at high concentrations, while at low concentrations it induces cell cycle arrest, stimulates reorganization of sub-nuclear protein localization and controls splicing of several types of mRNAs [43].

Sudemycins (FR901464), an inhibitor of splicing showed cytotoxic activity against tumor cells both in vivo and in vitro in xenograft models through targeting SF3b factor [46].

Pladienolide is a naturally occurring anti-tumor macrolide that inhibits the process of mRNA splicing. Pladienolide binds directly to spliceosome-associated protein 155 (SAP155, SF3b subunit 1) and the inhibitory activity is dose-dependent. Data suggested that SF3b factor is a potential anti-tumor drug target [47]. 
E7107 that targets the U2 small nuclear ribonucleoprotein (snRNP) subunit SF3b is a derivative of the pladienolide family. This product is in clinical trial and promising results have been achieved [48, 49].

Other synthetic or natural inhibitors of mRNA splicing are under investigation in pre-clinical and clinical evaluation.

\section{Targeting tumor cells by microRNAs}

MicroRNAs (miRNAs or miR) are a type of non-coding small RNA molecules (21-25 nucleotides in length), which control gene expression. Several function, including regulation of gene expression, tumor cells resistance to treatments and behave as tumor suppressor genes have been described [50]. Dysregulation of miRNAs can be associated with several diseases and is involved in a variety of pathophysiologies due to aberrant expression [51, 52].

MiRNAs are involved in tumor cells sensitivity to treatments. It has been shown that miR-7 sensitized NSCLC cancer cells to paclitaxel [53]. Overexpression of miR-7 increased the sensitivity of NSCLC cells to paclitaxel by suppressing cell proliferation and induced cell apoptosis, while the inhibition of miR-7 disrupted the anti-proliferative and pro-apoptotic effects of paclitaxel. MiRNA such as miR-203 have been shown to downregulate TLR4 and the downstream cytokines in dendritic cells [51]. MiR-30e promoted apoptosis of acute myeloid leukemia (AML) cells to imatinib treatment through regulation of the oncogenic BCR-ABL protein. MiRNA-105 has been demonstrated to inhibit cell proliferation and repressed PI3K/Akt signaling pathway in hepatocellular carcinoma [54].

Overexpression of miR-5481 inhibited NSCLC cell migration and invasion. MiR-5481 can bind to Akt1 and overexpression of Akt1 inverse the effects of miR-5481 in NSCLC cells. It is indicated that Akt1 is involved in the effects of miR-5481 and suppresses the migration and invasion of NSCLC cells [55].

Conversely, some miRNAs are involved in tumor cells resistance to different therapeutic agents. Overexpression of miR-1, miR-125a, miR-150, and miR-425 in glioblastoma increased the resistance of tumor cells to radiotherapy via upregulation of the cell cycle checkpoint response. Antagonists of these miRNAs sensitized glioblastoma cells to irradiation, suggesting their potential as targets for preventing therapeutic resistance [56]. 


\section{Monoclonal antibodies: the most specific tools for targeted cancer therapy}

Extracellular molecules such as cell surface receptors or soluble proteins are the conventional targets for mAbs. Several cluster of differentiation (CD) markers such as CD20, CD23, CD33, CD40, CD52, CD74, CD152 [cytotoxic T lymphocyte antigen-4 (CTLA-4)], CD279 [programmed death-1 (PD-1)], and CD274 [programmed death-ligand 1 (PD-L1)] are appropriate targets, which are under investigation for TBCT by mAbs (Table 3). MAbs against these molecules destroy tumor cells by different mechanisms such as complementdependent cytotoxicity (CDC), antibody-dependent cell mediated lysis (ADCC) and induction of direct apoptosis or necrosis [57-59]. MAbs targeting RTKs and several CD markers have been described in several articles and will not be discussed here; however, anti-CD20, antiCD52, anti-CD152, anti-CD279, and anti-CD274 mAbs are described briefly as interesting tools for targeted cancer therapy.

\section{Anti-CD20 mAb}

CD20 is a signature B-cell differentiation marker and is an activated-glycosylated phosphoprotein expressed on all B cells beginning at the pro-B stage $\left(\mathrm{CD} 45 \mathrm{R}^{+}, \mathrm{CD} 117^{+}\right)$with increased expression on mature $B$ cells [60]. This antigen is expressed in several malignancies, including CLL, B-cell lymphomas, hairy cell leukemia, Hodgkins disease, melanoma cancer stem cells, myeloma, and thymoma [61].

Currently, there are two types of anti-CD20 mAbs that were approved for the treatment of Bcell malignancies [62]. Rituximab (Rituxan) is a chimeric type I anti-CD20 mAb. This antibody is used as single agent or combination therapy in relapsed or refractory indolent-non Hodgkins lymphoma (NHL) [63] and CLL patients [64]. Rituximab exerts its cytotoxicity through CDC, ADCC and week direct apoptosis [65]. This antibody has become part of standard chemoimmunotherapy [(fludarabine, cyclophosphamide and rituximab (FCR)] for most of untreated CLL patients [66].

Ofatumumab (Arzerra) was the second anti-CD20 mAb developed after rituximab for cancer treatment. It is a humanized type I anti-CD20 mAb targeting a different epitope on CD20 than the one targeted by rituximab and demonstrated higher activity in CDC and ADCC compared to rituximab, in vitro [62]. It was approved on October 20, 2011, for the treatment of CLL patients who are refractory to alemtuzumab and fludarabine treatment $[65,67,68]$. Recently, 
(April 17, 2014) the FDA approved this mAbs as single agent therapy for the treatment of CLL patients with no prior treatment or for those who are not eligible for chemotherapy (fludarabine-based therapy).

Obinutuzumab (Gazyva) is a novel, third generation fully humanized anti-CD20 mAb (type II). The Fc-region of obinutuzumab is glycol-engineered to result in higher affinity binding to the CD20 [69]. The mechanism of action of obinutuzumab is CDC and ADCC. Obinutuzumab showed an elevated ADCC as well as a markedly higher induction of direct cell death in vitro, compared to rituximab [186]. This mAb is able to elicit actin-dependent, lysosomal cell necrosis in CLL cells in vitro [187]. Obinutuzumab was approved by the FDA on November 1, 2013, for the treatment of CLL in combination with chemotherapy in previously untreated patients [70].

Currently, other anti-CD20 mAbs are in pre-clinical and clinical trials development.

\section{Anti-CD52 mAb (Alemtuzumab)}

Alemtuzumab is a humanized anti-CD52 mAb for the treatment of B-cell malignancies [71]. This mAb was approved on May 7, 2001 for the treatment of refractory CLL patients [72, 73].

The mechanism of action is mostly through ADCC and CDC [74, 75]. Alemtuzumab has serious side effects due to the widespread expression of CD52, including prolonged lymphopenia with an increased risk of infections [76]. About $20 \%$ of CLL patients have been shown to have cytomegalovirus (CMV) reactivation usually occurs after 3-8 weeks of alemtuzumab treatment [77]. This antibody has also been tested with limited success in the treatment of NHL and for the preparation of patients with blood malignancies for BM transplantation. There are also clinical trials ongoing to test the ability of this antibody to prevent tissue rejection in transplantation $[78,79]$.

\section{Anti-CTLA-4, PD-1/PD-L1 mAbs}

These molecules are involved in suppressing the immune system during different situations such as cancer. Targeting CTLA-4, PD-1/ PD-L1 antigens with mAbs has shown promising therapeutic results in several malignancies [80].

Several mAbs have been produced against these antigens, which are in preclinical and clinical settings for the treatment of various tumors, however; ipilimumab (anti-CTLA-4) and 
pembrolizumab (anti-PD-1) (Table 3) have been approved for the treatment of advanced melanoma on March 25, 2011 and September 4, 2014, respectively [80].

Ipilimumab is a fully human mAb that prevents CTLA-4 engagement and induce the activation of anti-tumor T-cell immune responses. Targeting CTLA-4 is currently the main immunotherapeutic approach that has shown significant clinical benefit in melanoma patients [81].

Pembrolizumab is a blocking humanized mAb (IgG4) that binds to the PD-1 and inhibits its interaction with PD-L1 and PD-L2, leading to the activation of immune response.

Currently these two mAbs are under clinical investigation for the treatment of several malignancies, including NSCLC, small cell lung cancer (SCLC), prostate, bladder, and metastatic hormone-refractory cancers [82-84].

There are other approved mAbs which are using as the first- or second-line of cancer treatment, including CD74 (milatuzumab), CD40 (dacetuzumab), CEA (labetuzumab), and CD23 (lumiliximab) molecules (Table 3). Moreover, several other humanized mAbs are in various stages of clinical testing but not yet approved by the authorities to be used for therapy.

\section{Targeting EMT in cancer}

The epithelial to mesenchymal transition (EMT) is involved in many processes, including tissue and organogenesis as well as metastatic spread of cancer cells. Targeting this phenomenon by preventing the transition of epithelial to mesenchymal cells might be a proper strategy. EMT is classified into 3 types. Type 1 EMT is the process of embryogenesis during the embryo development, type 2 refers to the normal process of wound healing and the process of cancer metastasis is classified as type 3. Loss of epithelial cell to cell junctions and apical-basal polarity are the major hallmarks of these three types [85].

Different intermediates such as transcription factors are responsible for EMT transition. The main regulators of EMT transition are transcription factors that are classified into 3 families, including zinc-finger E-box-binding (ZEB), TWIST and SNAIL. SNAIL2, ZEB1, ZEB2, E47, KLF8, TWIST1, and FOXC2 transcription factors promote EMT in various cancer cells $[86,87]$. 
The basic feature of EMT is the suppression of E-cadherin expression that is responsible for sustaining the cells junctions and cell-cell adhesion. SNAIL, TWIST and ZEB expression can suppress E-cadherin and activating critical mesenchymal genes, including $\mathrm{N}$-cadherin, vimentin and fibronectin. These transcription factors regulates and activates the expression of mesenchymal genes while inhibiting epithelial genes expression [85].

Several mechanisms have been suggested to target EMT process for TBCT. These EMT targets are transcriptional regulators such as SNAIL, mediators (e.g. TGF $\beta$ ), non-coding RNAs, and cancer stem cells (CSCs). Moreover, targeting the tumor microenvironment interactions, the role in initiation and termination of EMT might be considered [85].

Various inhibitors, including CX-4945, EW-7195, EW-7197, IN-1130, SB-431542, SD-208, SD-093, LY580276, LY-573636, and LY2152799 are among EMT inhibitors [88]. These drugs target ALK5 (or TGF $\beta$ type 1 receptor) kinase. Ligation of TGF $\beta$ receptors (type 1 and 2) by TGF $\beta$ will ultimately activate Smad proteins and their translocation to the nucleus. In the nucleus, Smad proteins regulate the expression of target genes including those involved in EMT, therefore, blocking ALK5 by theses inhibitors has demonstrated promises in inhibiting EMT [89].

\section{Immune modulatory (IMiD) agents and targeted therapy}

It has been shown that several types of chemotherapy agents have side effects on immune cells. Therefore, a special class of therapeutic agents called immunomodulatory (IMiDs) agents was developed to be used in combination with chemotherapy or other targeted therapies to prevent immune system suppression. Later on, several groups showed that some of these drugs have not only immunomodulatory effects, but also can directly kill tumor cells.

Currently, a few IMiDs agents have been approved by the FDA for the treatment of B-cell malignancies and several others are in pre-clinical or clinical settings. Lenalidomide and ibrutinib belong to this group [90]. 


\section{Lenalidomide}

Lenalidomide or revlimid is a derivative of thalidomide and has several mechanisms of action. The anti-tumor and immunomodulatory effects are mediated through regulating innate and specific immune responses. For instance, it changed the immunological profile of the tumor cells microenvironment by preventing the secretion of pro-survival cytokines such as TNF $\alpha$, IL-1 $\beta$ and IL-6, while favoring that of IL-2, IL-10, IL-12, and interferon $\gamma$ (IFN $\gamma$ ) [91]. Moreover, it activated T and NK cells, inhibited tumor angiogenesis [92-94], changed the balance of Th1/Th2 cell toward Th1, increased the expression of CD80, CD86, HLA-DR, and stimulated the cytotoxic effects of T lymphocytes and natural killer cells [95].

Lenalidomide is mostly administrated for the treatment of patients with relapsed or refractory CLL [96, 97], multiple myeloma [98], MCL [99], and a few other lymphomas [91, 100]. The mechanism of action of lenalidomide exerts direct cell cycle arresting and pro-apoptotic effects on cancer cells, interrupts with physical and functional communication with the tumor microenvironment and mediates immunostimulatory activity. The cell cycle arrest and the consequent anti-tumor effects of lenalidomide are through the upregulation of cyclindependent kinase inhibitors (CDKNs) [101].

Lenalidomide inhibited the immunosuppressive effects of myeloid-derived suppressor cells (MDSCs) and regulatory $\mathrm{T}$ cells by preventing the expression of the transcription factor Forkhead box P3 (FOXP3). Indeed, this IMiD has shown robust anti-neoplastic effects in multiple myeloma patients previously subjected to stem cell transplantation while stimulating a transient increase in $\mathrm{CD} 4^{+} \mathrm{FOXP} 3^{+}$Tregs [102].

\section{Ibrutinib}

Ibrutinib (Imbruvica) is an inhibitor of Bruton tyrosine kinase (Btk) that was reported in 2007 [103]. This inhibitor was developed from the PCI-29732 inhibitor [103]. It binds covalently with cysteine (Cys) 481 in the ATP-binding pocket of Btk.

Ibrutinib binds to the non-phosphorylated Btk and stabilizes this inactive conformation by internalizing Tyr 551 and prevents its phosphorylation. Ibrutinib inhibits other kinases, including Blk, Bmx, EGFR, Itk, and JAK3 [104]. These kinases have a cysteine residue in the homologous location to Btk. Ibrutinib has shown to be 1000-fold more selective for inhibition of BCR signaling in B cells over TCR signaling in T cells [104, 105]. 
Currently, several trials are assessing ibrutinib in malignant disorders, including CLL, DLBCL and Waldenström's macroglobulinemia, alone or in combination with other drugs [106].

Recent studies have showed that ibrutinib blocked IL-2 inducible tyrosine kinase (Itk) in T cells. Th1 cells; however, express another kinase called resting lymphocyte kinase (Rlk or Txk). Following ibrutinib treatment, Itk in Th cells is inhibited and only Th1 cells survived due to the activation of Rlk survival pathway [107]. This event changes the balance of Th1/Th2 toward Th1 cells that are the main cells activating immune cells against tumor cells, intracellular pathogens and preventes the production of autoreactive antibodies [107].

\section{Targeting post-translational modification of proteins}

Post-translational modification (PTM) of proteins by glycosylation, phosphorylation, acetylation, ubiquitination, and other modifications is essential in moderating protein function. Aberrant PTMs underlie a majority of human diseases, including cancer and now it is well established that altered modifications vary significantly for cancer cells compared to normal counterparts and each type of tumor might have a unique PTM signature [108]. Current development of analytical techniques and instrumentation, especially in mass spectrometry has made it possible to recognize the type of protein PTMs in normal and cancer cells [109]. However, there are several issues that have not been solved such as determining the exact PMTs in tumor cells, mainly due to the intraclonal diversity of tumor cells within a population.

Generation of mAbs that target PTMs might be of high interest. However, due to the low immunogenicity of non-protein molecules, production of effective mAbs against the abovementioned molecules is a major challenge. Moreover, for production of therapeutic mAbs, more information regarding PTMs in the protein of interest might be necessary.

It has been shown that $\operatorname{IgM}$ anti-ganglioside antibodies induced by melanoma cell vaccine correlated with survival of melanoma patients [110, 111]. Numerous anti-disialoganglioside mAbs have also been developed for clinical use and have been trialed in metastatic melanoma. Disialoganglioside GD2 is overexpressed on the surface of tumors of neuroectodermal origin and is an interesting target for mAbs [112]. 
Targeting PTMs is in early stages and moreover, it is a challenging field and further investigations are warranted.

\section{Inhibition of Autophagy}

Autophagy process was first described by Porter KR et al. [113]. Autophagy is a catabolic activity involving the degradation of cell components through the lysosomal machinery. Several enzymes, including 30 autophagy-associated molecules (Atg) and 50 hydrolases within the lysosomes are involved in autophagy [114]. Cells use autophagy for the maintenance of cellular metabolism under starvation condition and to remove injured organelles under stress. This process is essential for normal growth control and is defective in several tumors as indicated as a pro-survival process in progressive tumor cells, leading to cancer resistance $[115,116]$.

Several pre-clinical and clinical trials are ongoing to develop therapeutic drugs to inhibit autophagy. Different inhibitors of autophagy are classified as early- or late-stage inhibitors. Inhibitors such as 3-Methyladenine (3-MA), wortmannin and LY294002 target the Vps34 (class III PI3K) and have been categorized as early-stage and chloroquine (CQ), HCQ, bafilomycin A1, and monensin that prevent the lysosomal function are classified as late-stage inhibitors [117]. Microtubule disrupting drugs like taxanes, nocodazole, colchicine, and vinca are defined as a separate class of autophagy inhibitors. CQ, HCQ and quinacrine are testing in clinical trials as promising anti-autophagy inhibitors.

Moreover, it is known that autophagy process happens in minor population of tumor cells and these inhibitors may have better effects in combination with other anti-cancer agents. Indeed, most clinical trials have used HCQ in combination with other inhibitors. Autophagy inhibition can also improve the anti-tumor immune responses. Immunotherapeutic methods such as dendritic cell (DC) vaccines, adoptive transfer of $\mathrm{T}$ cells and administration of mAbs or cytokines are effective after the inhibition of the autophagic process [118]. 


\section{Targeting the hypoxia induction}

Hypoxia is a main feature of solid tumors, inducing an aggressive phenotype of tumors that is more resistant to therapies [119]. This process activates several pathways, including the hypoxia inducible factor (HIF), which mediates the effects of hypoxia in tumor tissues. Therefore, targeting the hypoxia by different inhibitors might be a proper treatment strategy [120].

HIF-1 inhibitors have been shown to decrease tumor cells proliferation, increase necrosis and apoptosis of the cells and reduce tumor cell resistance to conventional therapies [121].

As HIF-1 is part of a transcriptional complex, special strategies are necessary to target hypoxia by inhibiting the HIF-1. Antisense strategies have been shown to decrease the expression of HIF-1a [122] and using a dominant-negative HIF-1a has been shown to decrease tumorigenicity of cancer cells by inhibiting glucose metabolism [123, 124].

Targeting protein-protein interactions by inhibiting HIF-1a is another approach to block the activity of HIF-1 [125]. For example, HIF-1a requires the transcriptional coactivator p300/CBP. Chetomin is an inhibitor of HIF-1 that prevented its binding to p300. It has been shown that chetomin disrupted the structure of the $\mathrm{CH} 1$ domain of p300 and inhibited its interaction with HIF. Moreover, systemic administration of chetomin blocked hypoxiainducible transcription within tumors and inhibited tumor cell growth [126].

EZN-2968 is an antisense (16 nucleotide residues) of HIF-1a mRNA and reduces HIF-1a protein synthesis. In vitro studies showed that EZN-2968 inhibited tumor cell growth and downregulated HIF-1a-regulated genes. Furthermore, in vivo studies demonstrated decreased expression of HIF-1a mRNA in the livers of mice and anti-tumor activity in xenograft models of human prostate cancer [127]. EZN-2968 is under evaluation in patients with advanced solid tumors and potential effects were observed in metastatic renal cell carcinoma and hepatocellular carcinoma [128]. Several other agents such as Echinomycin (DNA intercalator) are under investigation in pre-clinical and clinical trials.

Hypoxic media might be used against tumor cells using prodrugs that will be activated in these situations. Tumor cell death has been known to increase by the use of bioreductive prodrugs from several years ago [129, 130]. These prodrugs are activated under reductive conditions that are found within the tumor hypoxic environments. In most situations, it interferes with DNA replication and lead to cell death [35]. The ability for these prodrugs to 
increase the killing effects of both irradiation and chemotherapy make them potential agents in the treatment of solid tumors [131]. Several prodrugs have shown promising results in combination with radiotherapy [132].

Inducible nitric oxide synthase enzyme (iNOS) catalyzes and activates prodrugs under hypoxic situations and produces nitric oxide (NO). NO is also synthesized by other nitric oxide synthase enzyme [132]. NO that is released by donor drugs increased radio-sensitivity of human tumor cells in hypoxic conditions in vitro and mimics the effect of $\mathrm{O}_{2}$ by fixation of radiation-induced DNA damage. Several studies have shown that NO has high anti-tumor activity in high concentrations. Therefore, these prodrugs can overcome radio-resistant tumors [133]. Some of these prodrugs will be activated in the hypoxic microenvironment of the tumors (bioreductive pro-dugs) [132].

\section{Induce tumor cells differentiation}

Differentiated cells have low or no proliferative and metastasis activities. The approach of differentiation therapy of cancer has been introduced many years ago. Several encouraging in vitro and in vivo results have been obtained; however, the only successful clinical application has been all-trans-retinoic acid (ATRA)-based therapy of acute promyelocytic leukemia (APL) [134]. Pathogenesis of APL is related with a chromosomal translocation that disrupted retinoic acid receptor a (RAR $\alpha)$ gene located on the short arm of chromosome17 (q21) and resulted in an arrest of the early stage of promyelocyte differentiation. ATRA induces differentiation of APL blast cells [134].

This approach is useful for targeting CSCs by using compounds that induce the differentiation of these cells, and therefore make them sensitive to other therapies. The main characteristic of CSCs is self-renewing and the capacity to differentiate to several cell populations. By inducing CSCs differentiation, cells will become more susceptible to anti-tumor therapy, and lose their ability to rebuild the tumor later. As described 37 years ago, retinoic acid (RA) is an appropriate molecule that induces cellular differentiation in embryonal carcinoma cell lines [135] through the upregulation of genes that promotes differentiation, like $\alpha$-fetoprotein [136, 137] and downregulation of pluripotency-associated ones like Oct4 or telomerase [138].

Retinoic acid induces cell cycle arrest at the G1 stage through the downregulation of cyclin D1 by promoting protein degradation and suppressing mRNA synthesis as well as reduction 
of the phosphorylation of retinoblastoma $(\mathrm{Rb})$ protein [139]. RA has been demonstrated to induce cellular differentiation of keratinocytes, teratocarcinoma cells and APL, melanoma, and neuroblastoma cells in vitro [140-142]. Clinical studies have demonstrated some success, by combination of RA with other treatment protocols to overcome retinoid resistance [143]. In vitro studies have shown that combination of RA with HDAC inhibitors restores the expression of RAR $\beta 2$ by renal cancer cells in xenografts, followed by inhibition of tumor growth [144] as well as in breast and thyroid cancers [145, 146]. Combination of RA and HDACs inhibitors has therapeutic effects in leukemia patients [147]. 


\section{Conclusions}

Current data have demonstrated the high efficiency of TBCT agents and methods. Even the data are encouraging, however resistance to new agents, the plasticity of cancer cells, mutations, crosstalks between intracellular survival pathways and with the microenvironment, upregulation of other oncogenes, the tumor heterogeneity and cancer stem cell resistance are of the most important obstacles in front of researchers. Therefore, new applications such as appropriate drug combinations, new generation of mAbs and different methods of TBCT may be necessary. Moreover, specific targeting of cancer stem cells might be important to prevent tumor cell resistance to current TBCT methods; however, more investigation on CSCs phenotype, function and homing places for each cancer type is necessary. The early identification of mechanisms of tumor cell resistance is also important to change the treatment strategies or combine it with other methods. Finally, a better understanding of molecular, genetic and epigenetic factors involving in the pathogenesis of cancer are warranted. 


\section{Competing interest}

The author has no relevant affiliation or financial involvement with any organization or entity with a financial interest in or financial conflict with the subject matter or materials discussed in the manuscript.

Acknowledgements: I thank professor Hakan Mellstedt for his excellent support. This study was supported by a grant from Felix Mindus foundation. 


\section{References:}

1. Zhou L, Xu N, Sun Y, Liu XM. Targeted biopharmaceuticals for cancer treatment. Cancer Lett. 2014; 352 (2): 145-51.

2. MacDonald V. Chemotherapy: managing side effects and safe handling. Can Vet J. 2009; 50 (6): 665-8.

3. Peng X, Zhang MQ, Conserva F, Hosny G, Selivanova G, Bykov VJ, et al. APR246/PRIMA-1MET inhibits thioredoxin reductase 1 and converts the enzyme to a dedicated NADPH oxidase. Cell Death Dis. 2013; 4: e881.

4. Yap TA, Popat S. Toward precision medicine with next-generation EGFR inhibitors in non-small-cell lung cancer. Pharmgenomics Pers Med. 2014; 7: 285-95.

5. Hojjat-Farsangi M. Small-molecule inhibitors of the receptor tyrosine kinases: promising tools for targeted cancer therapies. Int J Mol Sci. 2014; 15 (8): 13768-801.

6. Serviss JT, Johnsson P, Grander D. An emerging role for long non-coding RNAs in cancer metastasis. Front Genet. 2014; 5: 234.

7. Grander D, Panaretakis T. Autophagy: cancer therapy's friend or foe? Future Med Chem. 2010; 2 (2): 285-97.

8. Grander D. How does interferon-alpha exert its antitumour activity in multiple myeloma? Acta Oncol. 2000; 39 (7): 801-5.

9. Hojjat-Farsangi M, Moshfegh A, Daneshmanesh AH, Khan AS, Mikaelsson E, Osterborg $A$, et al. The receptor tyrosine kinase ROR1 - An oncofetal antigen for targeted cancer therapy. Semin Cancer Biol. 2014; 29C: 21-31.

10. Li GN, Wang SP, Xue X, Qu XJ, Liu HP. Monoclonal antibody-related drugs for cancer therapy. Drug Discov Ther. 2013; 7 (5): 178-84.

11. Fauvel B, Yasri A. Antibodies directed against receptor tyrosine kinases: current and future strategies to fight cancer. MAbs. 2014; 6 (4): 838-51.

12. Ghoreschi K, Gadina M. Jakpot! New small molecules in autoimmune and inflammatory diseases. Exp Dermatol. 2014; 23 (1): 7-11.

13. Wu X, Liu X, Koul S, Lee CY, Zhang Z, Halmos B. AXL kinase as a novel target for cancer therapy. Oncotarget. 2014; 5 (20): 9546-63.

14. Zhang H, Nimmer PM, Tahir SK, Chen J, Fryer RM, Hahn KR, et al. Bcl-2 family proteins are essential for platelet survival. Cell Death Differ. 2007; 14 (5): 943-51.

15. Wilson WH, O'Connor OA, Czuczman MS, LaCasce AS, Gerecitano JF, Leonard JP, et al. Navitoclax, a targeted high-affinity inhibitor of BCL-2, in lymphoid malignancies: a phase 1 doseescalation study of safety, pharmacokinetics, pharmacodynamics, and antitumour activity. Lancet Oncol. 2010; 11 (12): 1149-59.

16. Rudin CM, Hann CL, Garon EB, Ribeiro de Oliveira M, Bonomi PD, Camidge DR, et al. Phase II study of single-agent navitoclax (ABT-263) and biomarker correlates in patients with relapsed small cell lung cancer. Clin Cancer Res. 2012; 18 (11): 3163-9.

17. Gobessi S, Laurenti L, Longo PG, Carsetti L, Berno V, Sica S, et al. Inhibition of constitutive and BCR-induced Syk activation downregulates $\mathrm{Mcl}-1$ and induces apoptosis in chronic lymphocytic leukemia B cells. Leukemia. 2009; 23 (4): 686-97.

18. Paterson A, Mockridge $\mathrm{Cl}$, Adams JE, Krysov S, Potter KN, Duncombe AS, et al. Mechanisms and clinical significance of BIM phosphorylation in chronic lymphocytic leukemia. Blood. 2012; 119 (7): 1726-36.

19. Smith DD, Goldstein L, Cheng M, James DF, Kunkel LA, Fardis M, et al. Modeling absolute lymphocyte counts after treatment of chronic lymphocytic leukemia with ibrutinib. Ann Hematol. 2014.

20. Tennant DA, Duran RV, Gottlieb E. Targeting metabolic transformation for cancer therapy. Nat Rev Cancer. 2010; 10 (4): 267-77. 
21.

Thomas HD, Calabrese CR, Batey MA, Canan S, Hostomsky Z, Kyle S, et al. Preclinical selection of a novel poly(ADP-ribose) polymerase inhibitor for clinical trial. Mol Cancer Ther. 2007; 6 (3): 945-56.

22. Plummer R, Jones $C$, Middleton $M$, Wilson R, Evans J, Olsen A, et al. Phase I study of the poly(ADP-ribose) polymerase inhibitor, AG014699, in combination with temozolomide in patients with advanced solid tumors. Clin Cancer Res. 2008; 14 (23): 7917-23.

23. Plummer R, Lorigan P, Steven N, Scott L, Middleton MR, Wilson RH, et al. A phase II study of the potent PARP inhibitor, Rucaparib (PF-01367338, AG014699), with temozolomide in patients with metastatic melanoma demonstrating evidence of chemopotentiation. Cancer Chemother Pharmacol. 2013; 71 (5): 1191-9.

24. Fong PC, Boss DS, Yap TA, Tutt A, Wu P, Mergui-Roelvink $M$, et al. Inhibition of poly(ADP-ribose) polymerase in tumors from BRCA mutation carriers. N Engl J Med. 2009; 361 (2): 123-34.

25. Richardson PG, Mitsiades CS, Laubach JP, Hajek R, Spicka I, Dimopoulos MA, et al. Preclinical data and early clinical experience supporting the use of histone deacetylase inhibitors in multiple myeloma. Leuk Res. 2013; 37 (7): 829-37.

26. Marquard L, Poulsen CB, Gjerdrum LM, de Nully Brown P, Christensen IJ, Jensen PB, et al. Histone deacetylase 1, 2, 6 and acetylated histone $\mathrm{H} 4$ in B- and T-cell lymphomas. Histopathology. 2009; 54 (6): 688-98.

27. Jazirehi AR. Regulation of apoptosis-associated genes by histone deacetylase inhibitors: implications in cancer therapy. Anticancer Drugs. 2010; 21 (9): 805-13.

28. West AC, Johnstone RW. New and emerging HDAC inhibitors for cancer treatment. J Clin Invest. 2014; 124 (1): 30-9.

29. Thaler F, Mercurio C. Towards selective inhibition of histone deacetylase isoforms: what has been achieved, where we are and what will be next. ChemMedChem. 2014; 9 (3): 523-6.

30. McGraw AL. Romidepsin for the treatment of T-cell lymphomas. Am J Health Syst Pharm. 2013; 70 (13): 1115-22.

31. Dong $M$, Ning ZQ, Xing PY, Xu JL, Cao HX, Dou GF, et al. Phase I study of chidamide (CS055/HBI-8000), a new histone deacetylase inhibitor, in patients with advanced solid tumors and lymphomas. Cancer Chemother Pharmacol. 2012; 69 (6): 1413-22.

32. Banerji U, van Doorn L, Papadatos-Pastos D, Kristeleit R, Debnam P, Tall M, et al. A phase I pharmacokinetic and pharmacodynamic study of CHR-3996, an oral class I selective histone deacetylase inhibitor in refractory solid tumors. Clin Cancer Res. 2012; 18 (9): 2687-94.

33. Dobashi $Y$, Watanabe $Y$, Miwa C, Suzuki S, Koyama S. Mammalian target of rapamycin: a central node of complex signaling cascades. Int J Clin Exp Pathol. 2011; 4 (5): 476-95.

34. Dancey JE, Monzon J. Ridaforolimus: a promising drug in the treatment of soft-tissue sarcoma and other malignancies. Future Oncol. 2011; 7 (7): 827-39.

35. Chen X, Zhao M, Hao M, Sun X, Wang J, Mao Y, et al. Dual inhibition of PI3K and mTOR mitigates compensatory AKT activation and improves tamoxifen response in breast cancer. Mol Cancer Res. 2013; 11 (10): 1269-78.

36. Laplante M, Sabatini DM. mTOR signaling in growth control and disease. Cell. 2012; 149 (2): 274-93.

37. Lebwohl D, Anak O, Sahmoud T, Klimovsky J, Elmroth I, Haas T, et al. Development of everolimus, a novel oral mTOR inhibitor, across a spectrum of diseases. Ann N Y Acad Sci. 2013; 1291: 14-32.

38. Molina AM, Motzer RJ, Heng DY. Systemic treatment options for untreated patients with metastatic clear cell renal cancer. Semin Oncol. 2013; 40 (4): 436-43.

39. Wan X, Shen N, Mendoza A, Khanna C, Helman LJ. CCl-779 inhibits rhabdomyosarcoma xenograft growth by an antiangiogenic mechanism linked to the targeting of mTOR/Hif-1alpha/VEGF signaling. Neoplasia. 2006; 8 (5): 394-401. 

hormone-targeted and HER2-targeted therapies in breast cancer. Clin Adv Hematol Oncol. 2013; 11 (4): 217-24.

41. Kabir A, Suresh Kumar G. Targeting double-stranded RNA with spermine, 1naphthylacetyl spermine and spermidine: a comparative biophysical investigation. J Phys Chem $B$. 2014; 118 (38): 11050-64.

42. Pan Q, Shai O, Lee LJ, Frey BJ, Blencowe BJ. Deep surveying of alternative splicing complexity in the human transcriptome by high-throughput sequencing. Nat Genet. 2008; 40 (12): 1413-5.

43. Pawellek A, McElroy S, Samatov T, Mitchell L, Woodland A, Ryder U, et al. Identification of Small Molecule Inhibitors of Pre-mRNA Splicing. J Biol Chem. 2014.

44. Thomas T, Balabhadrapathruni S, Gallo MA, Thomas TJ. Development of polyamine analogs as cancer therapeutic agents. Oncol Res. 2002; 13 (3): 123-35.

45. Casero RA, Jr., Marton LJ. Targeting polyamine metabolism and function in cancer and other hyperproliferative diseases. Nat Rev Drug Discov. 2007; 6 (5): 373-90.

46. Fan L, Lagisetti C, Edwards CC, Webb TR, Potter PM. Sudemycins, novel small molecule analogues of FR901464, induce alternative gene splicing. ACS Chem Biol. 2011; 6 (6): 582-9.

47. Kotake $Y$, Sagane K, Owa T, Mimori-Kiyosue $Y$, Shimizu $H$, Uesugi $M$, et al. Splicing factor SF3b as a target of the antitumor natural product pladienolide. Nat Chem Biol. 2007; 3 (9): 570-5.

48. Hong DS, Kurzrock R, Naing A, Wheler JJ, Falchook GS, Schiffman JS, et al. A phase I, open-label, single-arm, dose-escalation study of E7107, a precursor messenger ribonucleic acid (premRNA) splicesome inhibitor administered intravenously on days 1 and 8 every 21 days to patients with solid tumors. Invest New Drugs. 2014; 32 (3): 436-44.

49. Eskens FA, Ramos FJ, Burger H, O'Brien JP, Piera A, de Jonge MJ, et al. Phase I pharmacokinetic and pharmacodynamic study of the first-in-class spliceosome inhibitor E7107 in patients with advanced solid tumors. Clin Cancer Res. 2013; 19 (22): 6296-304.

50. Jayaraj GG, Nahar S, Maiti S. Nonconventional chemical inhibitors of microRNA: therapeutic scope. Chem Commun (Camb). 2014.

51. Zhou M, Chen J, Zhou L, Chen W, Ding G, Cao L. Pancreatic cancer derived exosomes regulate the expression of TLR4 in dendritic cells via miR-203. Cell Immunol. 2014; 292 (1-2): 65-9.

52. Caramuta S, Lee L, Ozata DM, Akcakaya P, Georgii-Hemming P, Xie H, et al. Role of microRNAs and microRNA machinery in the pathogenesis of diffuse large B-cell lymphoma. Blood Cancer J. 2013; 3: e152.

53. Liu R, Liu X, Zheng $Y$, Gu J, Xiong S, Jiang P, et al. MicroRNA-7 sensitizes non-small cell lung cancer cells to paclitaxel. Oncol Lett. 2014; 8 (5): 2193-200.

54. Shen G, Rong X, Zhao J, Yang X, Li H, Jiang H, et al. MicroRNA-105 suppresses cell proliferation and inhibits PI3K/AKT signaling in human hepatocellular carcinoma. Carcinogenesis. 2014.

55. Liu C, Yang $\mathrm{H}, \mathrm{Xu} \mathrm{Z}$, Li D, Zhou M, Xiao $\mathrm{K}$, et al. microRNA-548I is involved in the migration and invasion of non-small cell lung cancer by targeting the AKT1 signaling pathway. J Cancer Res Clin Oncol. 2014.

56. Moskwa P, Zinn PO, Choi YE, Shukla SA, Fendler W, Chen CC, et al. A Functional Screen Identifies miRs that Induce Radioresistance in Glioblastomas. Mol Cancer Res. 2014.

57. Hojjat-Farsangi M, Ghaemimanesh F, Daneshmanesh AH, Bayat AA, Mahmoudian J, Jeddi-Tehrani $M$, et al. Inhibition of the receptor tyrosine kinase ROR1 by anti-ROR1 monoclonal antibodies and siRNA induced apoptosis of melanoma cells. PLoS One. 2013; 8 (4): e61167.

58. Daneshmanesh AH, Hojjat-Farsangi M, Khan AS, Jeddi-Tehrani M, Akhondi MM, Bayat $A A$, et al. Monoclonal antibodies against ROR1 induce apoptosis of chronic lymphocytic leukemia (CLL) cells. Leukemia. 2012; 26 (6): 1348-55. 
59.

Staff C, Magnusson CG, Hojjat-Farsangi M, Mosolits S, Liljefors M, Frodin JE, et al. Induction of IgM, IgA and IgE antibodies in colorectal cancer patients vaccinated with a recombinant CEA protein. J Clin Immunol. 2012; 32 (4): 855-65.

60. Riley JK, Sliwkowski MX. CD20: a gene in search of a function. Semin Oncol. 2000; 27 (6 Suppl 12): 17-24.

61. Fang D, Nguyen TK, Leishear K, Finko R, Kulp AN, Hotz S, et al. A tumorigenic subpopulation with stem cell properties in melanomas. Cancer Res. 2005; 65 (20): 9328-37.

62. Bologna L, Gotti E, Da Roit F, Intermesoli T, Rambaldi A, Introna M, et al. Ofatumumab is more efficient than rituximab in lysing $B$ chronic lymphocytic leukemia cells in whole blood and in combination with chemotherapy. J Immunol. 2013; 190 (1): 231-9.

63. Fabbri A, Cencini E, Rigacci L, Bartalucci G, Puccini B, Dottori R, et al. Efficacy and safety of rituximab plus low-dose oral fludarabine and cyclophosphamide as first-line treatment of Elderly patients with indolent Non hodgkin lymphomas. Leuk Lymphoma. 2013.

64. Robak T. Rituximab for chronic lymphocytic leukemia. Expert Opin Biol Ther. 2012; 12 (4): 503-15.

65. Bauer K, Rancea M, Roloff V, Elter T, Hallek M, Engert A, et al. Rituximab, ofatumumab and other monoclonal anti-CD20 antibodies for chronic lymphocytic leukaemia. Cochrane Database Syst Rev. 2012; 11: CD008079.

66. Goteri G, Olivieri A, Ranaldi R, Lucesole M, Filosa A, Capretti R, et al. Bone marrow histopathological and molecular changes of small B-cell lymphomas after rituximab therapy: comparison with clinical response and patients outcome. Int J Immunopathol Pharmacol. 2006; 19 (2): 421-31.

67. Wierda WG, Kipps TJ, Mayer J, Stilgenbauer S, Williams CD, Hellmann A, et al. Ofatumumab as single-agent CD20 immunotherapy in fludarabine-refractory chronic lymphocytic leukemia. J Clin Oncol. 2010; 28 (10): 1749-55.

68. Smolej L, Doubek M, Panovska A, Simkovic M, Brychtova Y, Belada D, et al. Rituximab in combination with high-dose dexamethasone for the treatment of relapsed/refractory chronic lymphocytic leukemia. Leuk Res. 2012; 36 (10): 1278-82.

69. Abou-Nassar K, Brown JR. Novel agents for the treatment of chronic lymphocytic leukemia. Clin Adv Hematol Oncol. 2010; 8 (12): 886-95.

70. Goede V, Hallek M. Optimal pharmacotherapeutic management of chronic lymphocytic leukaemia: considerations in the elderly. Drugs Aging. 2011; 28 (3): 163-76.

71. McCoyd M. Update on therapeutic options for multiple sclerosis. Neurol Clin. 2013; 31 (3): 827-45.

72. Tsimberidou AM, Keating MJ. Treatment of fludarabine-refractory chronic lymphocytic leukemia. Cancer. 2009; 115 (13): 2824-36.

73. Osterborg A, Foa R, Bezares RF, Dearden C, Dyer MJ, Geisler C, et al. Management guidelines for the use of alemtuzumab in chronic lymphocytic leukemia. Leukemia. 2009; 23 (11): 1980-8.

74. Hale G. The CD52 antigen and development of the CAMPATH antibodies. Cytotherapy. 2001; 3 (3): 137-43.

75. Sorokin P. Campath-1H. Clin J Oncol Nurs. 2001; 5 (2): 65-6.

76. Ravandi F, O'Brien S. Alemtuzumab. Expert Rev Anticancer Ther. 2005; 5 (1): 39-51.

77. Karlsson C, Lundin J, Kimby E, Kennedy B, Moreton P, Hillmen P, et al. Phase II study of subcutaneous alemtuzumab without dose escalation in patients with advanced-stage, relapsed chronic lymphocytic leukaemia. Br J Haematol. 2009; 144 (1): 78-85.

78. Myrvang H. Transplantation: alemtuzumab induction is safe for renal transplant recipients. Nat Rev Nephrol. 2011; 7 (7): 362.

79. Hanaway MJ, Woodle ES, Mulgaonkar S, Peddi VR, Kaufman DB, First MR, et al. Alemtuzumab induction in renal transplantation. N Engl J Med. 2011; 364 (20): 1909-19.

80. Postow MA, Callahan MK, Wolchok JD. Immune Checkpoint Blockade in Cancer Therapy. J Clin Oncol. 2015. 

action in immune modulation. Curr Opin Oncol. 2014; 26 (2): 204-14.

82. Finkelstein SE, Salenius S, Mantz CA, Shore ND, Fernandez EB, Shulman J, et al. Combining Immunotherapy and Radiation for Prostate Cancer. Clin Genitourin Cancer. 2015; 13 (1): $1-9$.

83. Vasekar M, Liu X, Zheng H, Belani CP. Targeted immunotherapy for non-small cell lung cancer. World journal of clinical oncology. 2014; 5 (2): 39-47.

84. Langer LF, Clay TM, Morse MA. Update on anti-CTLA-4 antibodies in clinical trials. Expert Opin Biol Ther. 2007; 7 (8): 1245-56.

85. Kothari AN, Mi Z, Zapf M, Kuo PC. Novel clinical therapeutics targeting the epithelial to mesenchymal transition. Clin Transl Med. 2014; 3: 35.

86. Becker KF, Rosivatz E, Blechschmidt K, Kremmer E, Sarbia M, Hofler H. Analysis of the E-cadherin repressor Snail in primary human cancers. Cells Tissues Organs. 2007; 185 (1-3): 204-12.

87. Sanchez-Tillo E, Liu Y, de Barrios O, Siles L, Fanlo L, Cuatrecasas M, et al. EMTactivating transcription factors in cancer: beyond EMT and tumor invasiveness. Cell Mol Life Sci. 2012; 69 (20): 3429-56.

88. Arteaga CL. Inhibition of TGFbeta signaling in cancer therapy. Curr Opin Genet Dev. 2006; 16 (1): 30-7.

89. Fabregat I, Fernando J, Mainez J, Sancho P. TGF-beta signaling in cancer treatment. Curr Pharm Des. 2014; 20 (17): 2934-47.

90. Yang $Y$, Shaffer AL, 3rd, Emre NC, Ceribelli M, Zhang M, Wright G, et al. Exploiting synthetic lethality for the therapy of ABC diffuse large B cell lymphoma. Cancer Cell. 2012; 21 (6): 723-37.

91. Semeraro M, Vacchelli E, Eggermont A, Galon J, Zitvogel L, Kroemer G, et al. Trial Watch: Lenalidomide-based immunochemotherapy. Oncoimmunology. 2013; 2 (11): e26494.

92. LeBlanc R, Hideshima T, Catley LP, Shringarpure R, Burger R, Mitsiades N, et al. Immunomodulatory drug costimulates T cells via the B7-CD28 pathway. Blood. 2004; 103 (5): 178790.

93. Crane E, List A. Immunomodulatory drugs. Cancer Invest. 2005; 23 (7): 625-34.

94. Song W, van der Vliet HJ, Tai YT, Prabhala R, Wang R, Podar K, et al. Generation of antitumor invariant natural killer $\mathrm{T}$ cell lines in multiple myeloma and promotion of their functions via lenalidomide: a strategy for immunotherapy. Clin Cancer Res. 2008; 14 (21): 6955-62.

95. Harada T, Ozaki S, Oda A, Fujii S, Nakamura S, Miki H, et al. Association of Th1 and Th2 cytokines with transient inflammatory reaction during lenalidomide plus dexamethasone therapy in multiple myeloma. Int J Hematol. 2013; 97 (6): 743-8.

96. Gonzalez-Rodriguez AP, Payer AR, Acebes-Huerta A, Huergo-Zapico L, Villa-Alvarez M, Gonzalez-Garcia E, et al. Lenalidomide and Chronic Lymphocytic Leukemia. Biomed Res Int. 2013; 2013: 932010.

97. Ferrajoli A, Lee BN, Schlette EJ, O'Brien SM, Gao H, Wen S, et al. Lenalidomide induces complete and partial remissions in patients with relapsed and refractory chronic lymphocytic leukemia. Blood. 2008; 111 (11): 5291-7.

98. Kourelis TV, Kumar SK, Srivastava G, Gertz MA, Lacy MQ, Buadi FK, et al. Long term response to lenalidomide in patients with newly diagnosed multiple myeloma. Leukemia. 2013.

99. Song K, Herzog BH, Sheng M, Fu J, McDaniel JM, Ruan J, et al. Lenalidomide inhibits lymphangiogenesis in preclinical models of mantle cell lymphoma. Cancer Res. 2013.

100. Provencio M, Sanchez A, Sanchez-Beato M. New drugs and targeted treatments in Hodgkin's lymphoma. Cancer Treat Rev. 2013.

101. Alvarez-Fernandez S, Ortiz-Ruiz MJ, Parrott T, Zaknoen S, Ocio EM, San Miguel J, et al. Potent antimyeloma activity of a novel ERK5/CDK inhibitor. Clin Cancer Res. 2013; 19 (10): 2677-87.

102. Minnema MC, van der Veer MS, Aarts T, Emmelot M, Mutis T, Lokhorst HM. Lenalidomide alone or in combination with dexamethasone is highly effective in patients with 
relapsed multiple myeloma following allogeneic stem cell transplantation and increases the frequency of CD4+Foxp3+T cells. Leukemia. 2009; 23 (3): 605-7.

103. Pan Z, Scheerens H, Li SJ, Schultz BE, Sprengeler PA, Burrill LC, et al. Discovery of selective irreversible inhibitors for Bruton's tyrosine kinase. ChemMedChem. 2007; 2 (1): 58-61.

104. Honigberg LA, Smith AM, Sirisawad M, Verner E, Loury D, Chang B, et al. The Bruton tyrosine kinase inhibitor $\mathrm{PCl}-32765$ blocks B-cell activation and is efficacious in models of autoimmune disease and B-cell malignancy. Proc Natl Acad Sci U S A. 2010; 107 (29): 13075-80.

105. Xing L, Huang A. Bruton's TK inhibitors: structural insights and evolution of clinical candidates. Future Med Chem. 2014; 6 (6): 675-95.

106. Zheng $X$, Ding N, Song $Y$, Feng L, Zhu J. Different sensitivity of germinal center $B$ celllike diffuse large $B$ cell lymphoma cells towards ibrutinib treatment. Cancer Cell Int. 2014; 14 (1): 32.

107. Dubovsky JA, Beckwith KA, Natarajan G, Woyach JA, Jaglowski S, Zhong Y, et al. Ibrutinib is an irreversible molecular inhibitor of ITK driving a Th1-selective pressure in T lymphocytes. Blood. 2013; 122 (15): 2539-49.

108. Ohtsubo K, Marth JD. Glycosylation in cellular mechanisms of health and disease. Cell. 2006; 126 (5): 855-67.

109. Ruhaak LR, Miyamoto S, Lebrilla CB. Developments in the identification of glycan biomarkers for the detection of cancer. Mol Cell Proteomics. 2013; 12 (4): 846-55.

110. Takahashi T, Johnson TD, Nishinaka Y, Morton DL, Irie RF. IgM anti-ganglioside antibodies induced by melanoma cell vaccine correlate with survival of melanoma patients. J Invest Dermatol. 1999; 112 (2): 205-9.

111. Irie RF, Ollila DW, O'Day S, Morton DL. Phase I pilot clinical trial of human IgM monoclonal antibody to ganglioside GM3 in patients with metastatic melanoma. Cancer Immunol Immunother. 2004; 53 (2): 110-7.

112. Zhang S, Zhang HS, Cordon-Cardo C, Reuter VE, Singhal AK, Lloyd KO, et al. Selection of tumor antigens as targets for immune attack using immunohistochemistry: II. Blood group-related antigens. Int J Cancer. 1997; 73 (1): 50-6.

113. Ashford TP, Porter KR. Cytoplasmic components in hepatic cell lysosomes. J Cell Biol. 1962; 12: 198-202.

114. Lee J, Giordano S, Zhang J. Autophagy, mitochondria and oxidative stress: cross-talk and redox signalling. Biochem J. 2012; 441 (2): 523-40.

115. Zhang J, Yang Z, Xie L, Xu L, Xu D, Liu X. Statins, autophagy and cancer metastasis. Int J Biochem Cell Biol. 2013; 45 (3): 745-52.

116. Fullgrabe J, Heldring N, Hermanson O, Joseph B. Cracking the survival code: autophagy-related histone modifications. Autophagy. 2014; 10 (4): 556-61.

117. Viry E, Paggetti J, Baginska J, Mgrditchian T, Berchem G, Moussay E, et al. Autophagy: An adaptive metabolic response to stress shaping the antitumor immunity. Biochem Pharmacol. 2014.

118. Guan JL, Simon AK, Prescott M, Menendez JA, Liu F, Wang F, et al. Autophagy in stem cells. Autophagy. 2013; 9 (6): 830-49.

119. Burroughs SK, Kaluz S, Wang D, Wang K, Van Meir EG, Wang B. Hypoxia inducible factor pathway inhibitors as anticancer therapeutics. Future Med Chem. 2013; 5 (5): 553-72.

120. Ward C, Langdon SP, Mullen P, Harris AL, Harrison DJ, Supuran CT, et al. New strategies for targeting the hypoxic tumour microenvironment in breast cancer. Cancer Treat Rev. 2013; 39 (2): 171-9.

121. Moeller BJ, Dreher MR, Rabbani ZN, Schroeder T, Cao Y, Li CY, et al. Pleiotropic effects of HIF-1 blockade on tumor radiosensitivity. Cancer Cell. 2005; 8 (2): 99-110.

122. Yeo EJ, Chun YS, Park JW. New anticancer strategies targeting HIF-1. Biochem Pharmacol. 2004; 68 (6): 1061-9.

123. Poon E, Harris AL, Ashcroft M. Targeting the hypoxia-inducible factor (HIF) pathway in cancer. Expert Rev Mol Med. 2009; 11: e26. 

hypoxia-inducible factor-1 alpha reduces tumorigenicity of pancreatic cancer cells through the suppression of glucose metabolism. Am J Pathol. 2003; 162 (4): 1283-91.

125. Zinzalla G, Thurston DE. Targeting protein-protein interactions for therapeutic intervention: a challenge for the future. Future Med Chem. 2009; 1 (1): 65-93.

126. Kung AL, Zabludoff SD, France DS, Freedman SJ, Tanner EA, Vieira A, et al. Small molecule blockade of transcriptional coactivation of the hypoxia-inducible factor pathway. Cancer Cell. 2004; 6 (1): 33-43.

127. Greenberger LM, Horak ID, Filpula D, Sapra P, Westergaard M, Frydenlund HF, et al. A RNA antagonist of hypoxia-inducible factor-1alpha, EZN-2968, inhibits tumor cell growth. Mol Cancer Ther. 2008; 7 (11): 3598-608.

128. Jones DT, Harris AL. Small-molecule inhibitors of the HIF pathway and synthetic lethal interactions. Expert Opin Ther Targets. 2012; 16 (5): 463-80.

129. Connors TA, Whisson ME. Cure of mice bearing advanced plasma cell tumours with aniline mustard: the relationship between glucuronidase activity and tumour sensitivity. Nature. 1966; 210 (5038): 866-7.

130. Whisson ME, Connors TA. Cure of mice bearing advanced plasma cell tumours with aniline mustard. Nature. 1965; 206 (985): 689-91.

131. Bryant JL, Meredith SL, Williams KJ, White A. Targeting hypoxia in the treatment of small cell lung cancer. Lung Cancer. 2014; 86 (2): 126-32.

132. Ghattass K, Assah R, El-Sabban M, Gali-Muhtasib H. Targeting hypoxia for sensitization of tumors to radio- and chemotherapy. Curr Cancer Drug Targets. 2013; 13 (6): 670-85.

133. Wardman P, Rothkamm K, Folkes LK, Woodcock M, Johnston PJ. Radiosensitization by nitric oxide at low radiation doses. Radiat Res. 2007; 167 (4): 475-84.

134. Marchwicka A, Cebrat M, Sampath P, Sniezewski L, Marcinkowska E. Perspectives of differentiation therapies of acute myeloid leukemia: the search for the molecular basis of patients' variable responses to 1,25-dihydroxyvitamin $d$ and vitamin $d$ analogs. Front Oncol. 2014; 4: 125.

135. Strickland S, Mahdavi V. The induction of differentiation in teratocarcinoma stem cells by retinoic acid. Cell. 1978; 15 (2): 393-403.

136. Werneck MB. Endogenous anticancer mechanism: differentiation. Front Biosci (Schol Ed). 2012; 4: 1518-38.

137. Chen H, Dong JM, Liu Y, Chiu JF. Identification of a cis-acting element in the rat alphafetoprotein gene and its specific binding proteins in F9 cells during retinoic acid-induced differentiation. J Cell Biochem. 1999; 72 (1): 25-34.

138. Bunaciu RP, Yen A. Activation of the aryl hydrocarbon receptor AhR Promotes retinoic acid-induced differentiation of myeloblastic leukemia cells by restricting expression of the stem cell transcription factor Oct4. Cancer Res. 2011; 71 (6): 2371-80.

139. Tang $\mathrm{XH}$, Gudas LJ. Retinoids, retinoic acid receptors, and cancer. Annu Rev Pathol. 2011; 6: 345-64.

140. Fuchs E, Green H. Regulation of terminal differentiation of cultured human keratinocytes by vitamin A. Cell. 1981; 25 (3): 617-25.

141. Matthay KK, Reynolds CP, Seeger RC, Shimada H, Adkins ES, Haas-Kogan D, et al. Longterm results for children with high-risk neuroblastoma treated on a randomized trial of myeloablative therapy followed by 13-cis-retinoic acid: a children's oncology group study. J Clin Oncol. 2009; 27 (7): 1007-13.

142. Warrell RP, Jr., Frankel SR, Miller WH, Jr., Scheinberg DA, Itri LM, Hittelman WN, et al. Differentiation therapy of acute promyelocytic leukemia with tretinoin (all-trans-retinoic acid). N Engl J Med. 1991; 324 (20): 1385-93.

143. Duong V, Rochette-Egly C. The molecular physiology of nuclear retinoic acid receptors. From health to disease. Biochim Biophys Acta. 2011; 1812 (8): 1023-31. 

the histone deacetylase inhibitor trichostatin a inhibit the proliferation of human renal cell carcinoma in a xenograft tumor model. Clin Cancer Res. 2005; 11 (9): 3558-66.

145. Farias EF, Petrie K, Leibovitch B, Murtagh J, Chornet MB, Schenk T, et al. Interference with $\operatorname{Sin} 3$ function induces epigenetic reprogramming and differentiation in breast cancer cells. Proc Natl Acad Sci U S A. 2010; 107 (26): 11811-6.

146. Tavares TS, Nanus D, Yang XJ, Gudas LJ. Gene microarray analysis of human renal cell carcinoma: the effects of HDAC inhibition and retinoid treatment. Cancer Biol Ther. 2008; 7 (10): 1607-18.

147. Garcia-Manero G, Kantarjian HM, Sanchez-Gonzalez B, Yang H, Rosner G, Verstovsek S, et al. Phase $1 / 2$ study of the combination of 5-aza-2'-deoxycytidine with valproic acid in patients with leukemia. Blood. 2006; 108 (10): 3271-9. 